\title{
Existence and Uniqueness of Smooth Positive Solutions to a Class of Singular $m$-Point Boundary Value Problems
}

\author{
Xinsheng Du and Zengqin Zhao \\ School of Mathematics Sciences, Qufu Normal University, Qufu, Shandong 273165, China \\ Correspondence should be addressed to Xinsheng Du, duxinsheng@mail.qfnu.edu.cn \\ Received 2 April 2009; Revised 15 September 2009; Accepted 23 November 2009 \\ Recommended by Donal O'Regan \\ This paper investigates the existence and uniqueness of smooth positive solutions to a class of \\ singular m-point boundary value problems of second-order ordinary differential equations. A \\ necessary and sufficient condition for the existence and uniqueness of smooth positive solutions is \\ given by constructing lower and upper solutions and with the maximal theorem. Our nonlinearity \\ $f(t, u, v)$ may be singular at $v, t=0$ and /or $t=1$.
}

Copyright $@ 2009$ X. Du and Z. Zhao. This is an open access article distributed under the Creative Commons Attribution License, which permits unrestricted use, distribution, and reproduction in any medium, provided the original work is properly cited.

\section{Introduction and the Main Results}

In this paper, we will consider the existence and uniqueness of positive solutions to a class of second-order singular $m$-point boundary value problems of the following differential equation:

$$
-u^{\prime \prime}(t)=f(t, u(t), u(t)), \quad t \in(0,1),
$$

with

$$
u(0)=\sum_{i=1}^{m-2} \alpha_{i} u\left(\eta_{i}\right), \quad u(1)=0,
$$

where $0<\alpha_{i}<1, i=1,2, \ldots, m-2,0<\eta_{1}<\eta_{2}<\cdots<\eta_{m-2}<1$, are constants, $\sum_{i=1}^{m-2} \alpha_{i}<$ $1, m \geq 3$, and $f$ satisfies the following hypothesis: 
(H) $f(t, u, v):(0,1) \times(0,+\infty) \times(0,+\infty) \rightarrow[0,+\infty)$ is continuous, nondecreasing on $u$, and nonincreasing on $v$ for each fixed $t \in(0,1)$, there exists a real number $b \in R^{+}$ such that for any $r \in(0,1)$,

$$
f(t, u, r v) \leq r^{-b} f(t, u, v), \quad \forall(t, u, v) \in(0,1) \times(0,+\infty) \times(0,+\infty),
$$

there exists a function $g:[1, \infty) \rightarrow(0,+\infty), g(l)<l$, and $g(l) / l^{2}$ is integrable on $(1,+\infty)$ such that

$$
f(t, l u, v) \leq g(l) f(t, u, v), \quad \forall(t, u, v) \in(0,1) \times(0,+\infty) \times(0,+\infty), l \in[1,+\infty) .
$$

Remark 1.1. (i) Inequality (1.3) implies

$$
f(t, u, c v) \geq c^{-b} f(t, u, v), \quad \text { if } c \geq 1 .
$$

Conversely, (1.5) implies (1.3).

(ii) Inequality (1.4) implies

$$
f(t, c u, v) \geq\left(g\left(c^{-1}\right)\right)^{-1} f(t, u, v), \quad \text { if } 0<c<1 .
$$

Conversely, (1.6) implies (1.4).

Remark 1.2. It follows from (1.3), (1.4) that

$$
f(t, u, u) \leq \begin{cases}g\left(\frac{u}{v}\right) f(t, v, v), & \text { if } u \geq v>0 \\ \left(\frac{v}{u}\right)^{b} f(t, v, v), & \text { if } v \geq u>0 .\end{cases}
$$

When $f(t, u)$ is increasing with respect to $u$, singular nonlinear $m$-point boundary value problems have been extensively studied in the literature, see [1-3]. However, when $f(t, u, v)$ is increasing on $u$, and is decreasing on $v$, the study on it has proceeded very slowly. The purpose of this paper is to fill this gap. In addition, it is valuable to point out that the nonlinearity $f(t, u, v)$ may be singular at $t=0, t=1$ and / or $v=0$.

When referring to singularity we mean that the functions $f$ in (1.1) are allowed to be unbounded at the points $v=0, t=0$, and / or $t=1$. A function $u(t) \in C[0,1] \cap C^{2}(0,1)$ is called a $C[0,1]$ (positive) solution to (1.1) and (1.2) if it satisfies (1.1) and (1.2) $(u(t)>0$, for $t \in$ $(0,1))$. A $C[0,1]$ (positive) solution to (1.1) and (1.2) is called a smooth (positive) solution if $u^{\prime}\left(0^{+}\right)$and $u^{\prime}\left(1^{-}\right)$both exist $(u(t)>0$ for $t \in(0,1))$. Sometimes, we also call a smooth solution a $C^{1}[0,1]$ solution. It is worth stating here that a nontrivial $C[0,1]$ nonnegative solution to the problem (1.1), (1.2) must be a positive solution. In fact, it is a nontrivial concave function satisfying (1.2) which, of course, cannot be equal to zero at any point $t \in(0,1)$.

To seek necessary and sufficient conditions for the existence of solutions to the above problems is important and interesting, but difficult. Thus, researches in this respect are rare 
up to now. In this paper, we will study the existence and uniqueness of smooth positive solutions to the second-order singular $m$-point boundary value problem (1.1) and (1.2). A necessary and sufficient condition for the existence of smooth positive solutions is given by constructing lower and upper solutions and with the maximal principle. Also, the uniqueness of the smooth positive solutions is studied.

A function $\alpha(t)$ is called a lower solution to the problem (1.1), (1.2), if $\alpha(t) \in C[0,1] \cap$ $C^{2}(0,1)$ and satisfies

$$
\begin{gathered}
\alpha^{\prime \prime}(t)+f(t, \alpha(t), \alpha(t)) \geq 0, \quad t \in(0,1), \\
\alpha(0)-\sum_{i=1}^{m-2} \alpha_{i} \alpha\left(\eta_{i}\right) \leq 0, \quad \alpha(1) \leq 0 .
\end{gathered}
$$

Upper solution is defined by reversing the above inequality signs. If there exist a lower solution $\alpha(t)$ and an upper solution $\beta(t)$ to problem (1.1), (1.2) such that $\alpha(t) \leq \beta(t)$, then $(\alpha(t), \beta(t))$ is called a couple of upper and lower solution to problem (1.1), (1.2).

To prove the main result, we need the following maximal principle.

Lemma 1.3 (maximal principle). Suppose that $0<\eta_{1}<\eta_{2}<\cdots<\eta_{m-2}<b_{n}<1, n=1,2, \ldots$, and $F_{n}=\left\{u(t) \in C\left[0, b_{n}\right] \cap C^{2}\left(0, b_{n}\right), u(0)-\sum_{i=1}^{m-2} \alpha_{i} u\left(\eta_{i}\right) \geq 0, u\left(b_{n}\right) \geq 0\right\}$. If $u \in F_{n}$ such that $-u^{\prime \prime}(t) \geq 0, t \in\left(0, b_{n}\right)$ then $u(t) \geq 0, t \in\left[0, b_{n}\right]$.

Proof. Let

$$
\begin{gathered}
-u^{\prime \prime}(t)=\delta(t), \quad t \in\left(0, b_{n}\right), \\
u(0)-\sum_{i=1}^{m-2} \alpha_{i} u\left(\eta_{i}\right)=r_{1}, \quad u\left(b_{n}\right)=r_{2},
\end{gathered}
$$

then $r_{1} \geq 0, r_{2} \geq 0, \delta(t) \geq 0, t \in\left(0, b_{n}\right)$.

By integrating (1.9) twice and noting (1.10), we have

$$
\begin{aligned}
u(t)= & \frac{1}{b_{n}\left(1-\sum_{i=1}^{m-2} \alpha_{i}\right)+\sum_{i=1}^{m-2} \alpha_{i} \eta_{i}}\left[\left(\left(1-\sum_{i=1}^{m-2} \alpha_{i}\right) t+\sum_{i=1}^{m-2} \alpha_{i} \eta_{i}\right) r_{2}+\left(b_{n}-t\right) r_{1}\right] \\
& +\int_{0}^{b_{n}} G_{n}(t, s) \delta(s) d s+\frac{b_{n}-t}{b_{n}\left(1-\sum_{i=1}^{m-2} \alpha_{i}\right)+\sum_{i=1}^{m-2} \alpha_{i} \eta_{i}} \sum_{i=1}^{m-2} \alpha_{i} \int_{0}^{b_{n}} G_{n}\left(\eta_{i}, s\right) \delta(s) d s,
\end{aligned}
$$

where

$$
G_{n}(t, s)=\frac{1}{b_{n}} \begin{cases}t\left(b_{n}-s\right), & 0 \leq t \leq s \leq b_{n} \\ s\left(b_{n}-t\right), & 0 \leq s \leq t \leq b_{n}\end{cases}
$$


In view of (1.11) and the definition of $G_{n}(t, s)$, we can obtain $u(t) \geq 0, t \in\left[0, b_{n}\right]$. This completes the proof of Lemma 1.3.

Now we state the main results of this paper as follows.

Theorem 1.4. Suppose that $(H)$ holds, then a necessary and sufficient condition for the problem (1.1) and (1.2) to have smooth positive solution is that

$$
0<\int_{0}^{1} f(s, 1-s, 1-s) d s<\infty
$$

Theorem 1.5. Suppose that $(H)$ and (1.13) hold, then the smooth positive solution to problem (1.1) and $(1.2)$ is also the unique $C[0,1]$ positive solution.

\section{Proof of Theorem 1.4}

\subsection{The Necessary Condition}

Suppose that $w(t)$ is a smooth positive solution to the boundary value problem (1.1) and (1.2). We will show that (1.13) holds.

It follows from

$$
w^{\prime \prime}(t)=-f(t, w(t), w(t)) \leq 0, \quad t \in(0,1),
$$

that $w^{\prime}(t)$ is nonincreasing on $[0,1]$. Thus, by the Lebesgue theorem, we have

$$
\int_{0}^{1} f(t, w(t), w(t)) d t=-\int_{0}^{1} w^{\prime \prime}(t) d t=w^{\prime}(0+)-w^{\prime}(1-)<+\infty
$$

It is well known that $w(t)$ can be stated as

$$
\begin{aligned}
w(t)= & \int_{0}^{1} G(t, s) f(s, w(s), w(s)) d s \\
& +\frac{1-t}{\left(1-\sum_{i=1}^{m-2} \alpha_{i}\right)+\sum_{i=1}^{m-2} \alpha_{i} \eta_{i}} \sum_{i=1}^{m-2} \alpha_{i} \int_{0}^{1} G\left(\eta_{i}, s\right) f(s, w(s), w(s)) d s,
\end{aligned}
$$

where

$$
G(t, s)= \begin{cases}t(1-s), & 0 \leq t \leq s \leq 1 \\ s(1-t), & 0 \leq s \leq t \leq 1\end{cases}
$$


By (2.3) and (1.2) we have

$$
\frac{1}{\left(1-\sum_{i=1}^{m-2} \alpha_{i}\right)+\sum_{i=1}^{m-2} \alpha_{i} \eta_{i}} \sum_{i=1}^{m-2} \alpha_{i} \int_{0}^{1} G\left(\eta_{i}, s\right) f(s, w(s), w(s)) d s=\sum_{i=1}^{m-2} \alpha_{i}\left[w\left(\eta_{i}\right)\right]
$$

therefore because of (2.3) and (2.5),

$$
w(t) \geq(1-t) \sum_{i=1}^{m-2} \alpha_{i}\left[w\left(\eta_{i}\right)\right], \quad t \in[0,1]
$$

Since $w(t)$ is a smooth positive solution to (1.1) and (1.2), we have

$$
w(t)=\int_{t}^{1}\left(-w^{\prime}(s)\right) d s \leq \max _{t \in[0,1]}\left|w^{\prime}(t)\right|(1-t), \quad t \in[0,1]
$$

Let $m=\sum_{i=1}^{m-2} \alpha_{i} w\left[\left(\eta_{i}\right)\right], M=\max _{t \in[0,1]}\left|w^{\prime}(t)\right|$. From (2.6), (2.7) it follows that

$$
m(1-t) \leq w(t) \leq M(1-t), \quad t \in[0,1]
$$

Without loss of generality we may assume that $0<m<1<M$. This together with the condition $(H)$ implies

$$
\begin{aligned}
\int_{0}^{1} f(s, 1-s, 1-s) d s & \leq \int_{0}^{1} f\left(s, \frac{1}{m} w(s), \frac{1}{M} w(s)\right) d s \\
& \leq g\left(\frac{1}{m}\right) M^{b} \int_{0}^{1} f(s, w(s), w(s)) d s<+\infty
\end{aligned}
$$
have

$$
f(t, w(t), w(t))=-w^{\prime \prime}(t) \not \equiv 0, \quad t \in(0,1)
$$

therefore, there exists a positive number $t_{0} \in(0,1)$ such that $f\left(t_{0}, w\left(t_{0}\right), w\left(t_{0}\right)\right)>0$. Obviously, $w\left(t_{0}\right)>0$ and $1-t_{0}>0$. It follows from (1.7) that

$$
0<f\left(t_{0}, w\left(t_{0}\right), w\left(t_{0}\right)\right) \leq \begin{cases}g\left(\frac{w\left(t_{0}\right)}{1-t_{0}}\right) f\left(t_{0}, 1-t_{0}, 1-t_{0}\right), & \text { if } w\left(t_{0}\right) \geq 1-t_{0} \\ \left(\frac{1-t_{0}}{w\left(t_{0}\right)}\right)^{b} f\left(t_{0}, 1-t_{0}, 1-t_{0}\right), & \text { if } w\left(t_{0}\right) \leq 1-t_{0} .\end{cases}
$$


Consequently $f\left(t_{0}, 1-t_{0}, 1-t_{0}\right)>0$, which implies that

$$
\int_{0}^{1} f(s, 1-s, 1-s) d s>0 .
$$

From (2.9) and (2.12) it follows that

$$
0<\int_{0}^{1} f(s, 1-s, 1-s)<+\infty
$$

which is the required inequality.

\subsection{The Existence of Lower and Upper Solutions}

Since $g(l) / l^{2}$ is integrable on $[1,+\infty)$, thus

$$
\lim _{l \rightarrow+\infty} \inf \frac{g(l)}{l}=0 .
$$

Otherwise, if $\lim _{l \rightarrow+\infty}$ inf $g(l) / l=m_{0}>0$, then there exists a real number $X>0$, such that $g(l) / l^{2} \geq m_{0} / 2 l$ when $l>X$, this contradicts with the condition that $g(l) / l^{2}$ is integrable on $[1,+\infty)$. By condition $(H)$ and $(2.14)$ we have

$$
\begin{gathered}
f(t, r u, v) \geq h(r) f(t, u, v), \quad r \in(0,1), \\
\lim _{r \rightarrow 0^{+}} \sup \frac{r}{h(r)}=\lim _{p \rightarrow+\infty} \sup \frac{p^{-1}}{h\left(p^{-1}\right)}=\lim _{p \rightarrow+\infty} \inf \frac{g(p)}{p}=0,
\end{gathered}
$$

where $h(r)=\left(g\left(r^{-1}\right)\right)^{-1}, r \in(0,1)$.

Suppose that (1.13) holds. Let

$$
\begin{aligned}
b(t)= & \int_{0}^{1} G(t, s) f(s, 1-s, 1-s) d s \\
& +\frac{1-t}{1-\sum_{i=1}^{m-2} \alpha_{i}+\sum_{i=1}^{m-2} \alpha_{i} \eta_{i}} \sum_{i=1}^{m-2} \alpha_{i} \int_{0}^{1} G\left(\eta_{i}, s\right) f(s, 1-s, 1-s) d s .
\end{aligned}
$$

Since by (1.13), (2.17) we obviously have

$$
b(t) \in C^{1}[0,1] \cap C^{2}(0,1), \quad b^{\prime \prime}(t)=-f(t, 1-t, 1-t), \quad t \in(0,1),
$$

and there exists a positive number $k<1$ such that

$$
k(1-t) \leq b(t) \leq \frac{1}{k}(1-t), \quad t \in[0,1]
$$


By (2.14) and (2.16) we see, if $0<l<k$ is sufficiently small, then

$$
h(l k)-l \geq 0, \quad g\left(\frac{1}{l k}\right)-\frac{1}{l} \leq 0
$$

Let

$$
H(t)=l b(t), \quad Q(t)=\frac{1}{l} b(t), \quad t \in[0,1]
$$

Then from (2.19) and (2.21) we have

$$
l k(1-t) \leq H(t) \leq 1-t \leq Q(t) \leq \frac{1}{l k}(1-t), \quad t \in[0,1]
$$

Consequently, with the aid of (2.20), (2.22) and the condition $(H)$ we have

$$
\begin{aligned}
H^{\prime \prime}(t)+f(t, H(t), H(t)) & \geq f(t, l k(1-t), 1-t)-l f(t, 1-t, 1-t) \\
& \geq[h(l k)-l] f(t, 1-t, 1-t) \geq 0 \\
Q^{\prime \prime}(t)+f(t, Q(t), Q(t)) & \leq f\left(t, \frac{1}{l k}(1-t), 1-t\right)-\frac{1}{l} f(t, 1-t, 1-t) \\
& \leq\left[g\left(\frac{1}{l k}\right)-\frac{1}{l}\right] f(t, 1-t, 1-t) \leq 0 .
\end{aligned}
$$

From (2.17), (2.21) it follows that

$$
\begin{array}{ll}
H(0) & =\sum_{i=1}^{m-2} \alpha_{i} H\left(\eta_{i}\right), \quad H(1)=0, \\
Q(0) & =\sum_{i=1}^{m-2} \alpha_{i} Q\left(\eta_{i}\right), \quad Q(1)=0
\end{array}
$$

therefore, (2.23)-(2.26) imply that $H(t), Q(t)$ are lower and upper solutions to the problem (1.1) and (1.2), respectively.

\subsection{The Sufficient Condition}

First of all, we define a partial ordering in $C[0,1] \cap C^{2}(0,1)$ by $u \leq v$, if and only if

$$
u(t) \leq v(t), \quad \forall t \in[0,1] .
$$


Then, we will define an auxiliary function. For all $u(t) \in C[0,1] \cap C^{2}(0,1)$,

$$
g(t, u(t))= \begin{cases}f(t, H(t), H(t)), & \text { if } u(t) \leq H(t), \\ f(t, u(t), u(t)), & \text { if } H(t) \leq u(t) \leq Q(t), \\ f(t, Q(t), Q(t)), & \text { if } u(t) \geq Q(t) .\end{cases}
$$

By the assumption of Theorem 1.4, we have that $g:(0,1) \times(-\infty,+\infty) \rightarrow[0,+\infty)$ is continuous.

Let $\left\{b_{n}\right\}$ be a sequence satisfying $\eta_{m-2}<b_{1}<\cdots<b_{n}<b_{n+1}<\cdots<1$, and $b_{n} \rightarrow 1$ as $n \rightarrow+\infty$, and let $\left\{r_{n}\right\}$ be a sequence satisfying

$$
H\left(b_{n}\right) \leq r_{n} \leq Q\left(b_{n}\right), \quad n=1,2, \ldots
$$

For each $n$, let us consider the following nonsingular problem:

$$
\begin{gathered}
-u^{\prime \prime}(t)=g(t, u(t)), \quad t \in\left[0, b_{n}\right], \\
u(0)-\sum_{i=1}^{m-2} \alpha_{i} u\left(\eta_{i}\right)=0, \quad u\left(b_{n}\right)=r_{n} .
\end{gathered}
$$

Obviously, it follows from the proof of Lemma 1.3 that problem (2.30) is equivalent to the integral equation

$$
\begin{aligned}
u(t)= & A_{n} u(t)=\frac{\left(\left(1-\sum_{i=1}^{m-2} \alpha_{i}\right) t+\sum_{i=1}^{m-2} \alpha_{i} \eta_{i}\right) r_{n}}{b_{n}\left(1-\sum_{i=1}^{m-2} \alpha_{i}\right)+\sum_{i=1}^{m-2} \alpha_{i} \eta_{i}}+\int_{0}^{b_{n}} G_{n}(t, s) g(s, u(s)) d s \\
& +\frac{b_{n}-t}{b_{n}\left(1-\sum_{i=1}^{m-2} \alpha_{i}\right)+\sum_{i=1}^{m-2} \alpha_{i} \eta_{i}} \sum_{i=1}^{m-2} \alpha_{i} \int_{0}^{b_{n}} G_{n}\left(\eta_{i}, s\right) g(s, u(s)) d s, \quad t \in\left[0, b_{n}\right],
\end{aligned}
$$

where $G_{n}(t, s)$ is defined in the proof of Lemma 1.3. It is easy to verify that $A_{n}: X_{n} \rightarrow$ $X_{n}=C\left[0, b_{n}\right]$ is a completely continuous operator and $A_{n}\left(X_{n}\right)$ is a bounded set. Moreover, $u \in C\left[0, b_{n}\right]$ is a solution to (2.30) if and only if $A_{n} u=u$. Using the Schauder's fixed point theorem, we assert that $A_{n}$ has at least one fixed point $u_{n} \in C^{2}\left[0, b_{n}\right]$.

We claim that

$$
H(t) \leq u_{n}(t) \leq Q(t), \quad t \in\left[0, b_{n}\right]
$$

From this it follows that

$$
-u^{\prime \prime}(t)=f(t, u(t), u(t)), \quad t \in\left[0, b_{n}\right]
$$


Boundary Value Problems

Suppose by contradiction that $u_{n}(t) \leq Q(t)$ is not satisfied on $\left[0, b_{n}\right]$. Let

$$
z(t)=Q(t)-u_{n}(t), \quad t \in\left[0, b_{n}\right]
$$

therefore

$$
z\left(t^{*}\right)=\min _{t \in\left[0, b_{n}\right]} z(t)<0
$$

Since by the definition of $Q(t)$ and (2.30) we obviously have $t^{*} \neq 0, t^{*} \neq b_{n}$. Let

$$
\begin{aligned}
& c=\inf \left\{t_{1} \mid z(t)<0, t \in\left(t_{1}, t^{*}\right]\right\}, \\
& d=\sup \left\{t_{2} \mid z(t)<0, t \in\left[t^{*}, t_{2}\right)\right\} .
\end{aligned}
$$

So, when $t \in(c, d)$, we have $Q(t)<u_{n}(t)$, and

$$
\begin{aligned}
& g\left(t, u_{n}(t)\right)=f(t, Q(t), Q(t)), \\
& u_{n}^{\prime \prime}(t)+g(t, Q(t))=0, \\
& Q^{\prime \prime}(t)+g(t, Q(t))=Q^{\prime \prime}(t)+f(t, Q(t), Q(t)) \leq 0 .
\end{aligned}
$$

Therefore $z^{\prime \prime}(t)=Q^{\prime \prime}(t)-u_{n}^{\prime \prime}(t) \leq 0, t \in(c, d)$, that is, $z(t)$ is an upper convex function in $(c, d)$. By (2.30) and (2.36), for $c, d$ we have the following two cases:

(i) $z(c)=z(d)=0$,

(ii) $z(c)<0, z(d)=0$.

For case (i): it is clear that $z(t) \geq 0, t \in(c, d)$, this is a contradiction.

For case (ii): in this case $c=0, z^{\prime}\left(t^{*}\right)=0$. Since $z^{\prime}(t)$ is decreasing on $[c, d]$, thus, $z^{\prime}(t) \leq 0, t \in\left[t^{*}, d\right]$, that is, $z(t)$ is decreasing on $\left[t^{*}, d\right]$. From $z(d)=0$, we see $z\left(t^{*}\right) \geq 0$, which is in contradiction with $z\left(t^{*}\right)<0$.

From this it follows that $u_{n}(t) \leq Q(t), t \in\left[0, b_{n}\right]$.

Similarly, we can verify that $H(t) \leq u_{n}(t), t \in\left[0, b_{n}\right]$. Consequently (2.32) holds.

Using the method of [4] and [5, Theorem 3.2], we can obtain that there is a $C[0,1]$ positive solution $w(t)$ to (1.1), (1.2) such that $H(t) \leq w(t) \leq Q(t)$, and a subsequence of $\left\{u_{n}(t)\right\}$ converges to $w(t)$ on any compact subintervals of $(0,1)$. 


\section{Proof of Theorem 1.5}

Suppose that $u_{1}(t)$ and $u_{2}(t)$ are $C[0,1]$ positive solutions to (1.1) and (1.2), and at least one of them is a smooth positive solution. If $u_{1}(t) \not \equiv u_{2}(t)$ for any $t \in[0,1]$, without loss of generality, we may assume that $u_{2}\left(t^{*}\right)>u_{1}\left(t^{*}\right)$ for some $t^{*} \in(0,1)$. Let

$$
\begin{gathered}
T=\inf \left\{t_{1} \mid 0 \leq t_{1}<t^{*}, u_{2}(t)>u_{1}(t), t \in\left(t_{1}, t^{*}\right]\right\}, \\
S=\sup \left\{t_{2} \mid t^{*} \leq t_{2}<1, u_{2}(t)>u_{1}(t), t \in\left[t^{*}, t_{2}\right)\right\}, \\
y(t)=u_{1}(t) u_{2}^{\prime}(t)-u_{2}(t) u_{1}^{\prime}(t), \quad t \in(0,1) .
\end{gathered}
$$

It follows from (3.1) that

$$
0 \leq T<S \leq 1, \quad u_{2}(t) \geq u_{1}(t), \quad t \in(T, S) .
$$

By (1.2), it is easy to check that there exist the following two possible cases:

(1) $u_{1}(T)=u_{2}(T), u_{1}(S)=u_{2}(S)$,

(2) $u_{1}(T)<u_{2}(T), u_{1}(S)=u_{2}(S)$.

Assume that case (1) holds. By $u_{i}^{\prime \prime}(t) \leq 0$ on $(0,1)$, it is easy to see that $u_{i}^{\prime}(T+0) \quad(i=1,2)$ exist (finite or $\infty$ ), moreover, one of them must be finite. The same conclusion is also valid for $u_{i}^{\prime}(S-0)(i=1,2)$. It follows from (3.2) that

$$
\left.\left[u_{2}(t)-u_{1}(t)\right]\right|_{t=T+0} ^{\prime} \geq 0,
$$

consequently

$$
u_{2}^{\prime}(T+0) \geq u_{1}^{\prime}(T+0), \quad u_{1}^{\prime}(T+0) \text { is finite. }
$$

Similarly

$$
u_{2}^{\prime}(S-0) \leq u_{1}^{\prime}(S-0), \quad u_{1}^{\prime}(S-0) \text { is finite. }
$$

From (3.1), (3.4), and (3.5) we have

$$
\lim \inf _{t \rightarrow T+0} y(t) \geq 0 \geq \lim \sup _{t \rightarrow S-0} y(t) .
$$

On the other hand, (3.2), (1.7), and condition $(H)$ yield

$$
\begin{aligned}
f\left(t, u_{2}(t), u_{2}(t)\right) & \leq g\left(\frac{u_{2}(t)}{u_{1}(t)}\right) f\left(t, u_{1}(t), u_{1}(t)\right) \\
& \leq \frac{u_{2}(t)}{u_{1}(t)} f\left(t, u_{1}(t), u_{1}(t)\right), \quad t \in(T, S),
\end{aligned}
$$


that is,

$$
\frac{f\left(t, u_{2}(t), u_{2}(t)\right)}{u_{2}(t)} \leq \frac{f\left(t, u_{1}(t), u_{1}(t)\right)}{u_{1}(t)}, \quad t \in(T, S),
$$

therefore

$$
\frac{u_{1}^{\prime \prime}(t)}{u_{1}(t)} \leq \frac{u_{2}^{\prime \prime}(t)}{u_{2}(t)}, \quad t \in(T, S) .
$$

From this it follows that

$$
y^{\prime}(t)=u_{1}(t) u_{2}^{\prime \prime}(t)-u_{2}(t) u_{1}^{\prime \prime}(t) \geq 0, \quad t \in(T, S) .
$$

If $y^{\prime}(t) \equiv 0$ on $(T, S)$, then, by (3.6) we have $y(t) \equiv 0$, and then $\left(u_{2}(t) / u_{1}(t)\right)^{\prime} \equiv 0$, which imply that there exists a positive number $c$ such that $u_{2}(t)=c u_{1}(t)$ on $(T, S)$. It follows from (3.2) that $c>1$, therefore $T=0, S=1$. Substituting $u_{2}(t)=c u_{1}(t)$ into (1.1) and using condition $(H)$, we have

$$
\begin{aligned}
c f\left(t, u_{1}(t), u_{1}(t)\right) & =f\left(t, c u_{1}(t), c u_{1}(t)\right) \\
& \leq g(c) f\left(t, u_{1}(t), u_{1}(t)\right), \quad t \in(0,1) .
\end{aligned}
$$

Noticing (3.11) and $f\left(t, u_{1}(t), u_{1}(t)\right) \not \equiv 0, t \in(0,1)$, we have

$$
c \leq g(c),
$$

which contradicts with the condition that $g(c)<c$. Therefore, $y^{\prime}(t) \geq 0$ and $y^{\prime}(t) \not \equiv 0$ on $(T, S)$. Thus, $y(T+0)<y(S-0)$, which contradicts with (3.6). So case (1) is impossible.

By analogous methods, we can obtain a contradiction for case (2). So $u_{1}(t) \equiv u_{2}(t)$ for any $t \in[0,1]$, which implies that the result of Theorem 1.5 holds.

\section{Concerned Remarks and Applications}

Remark 4.1. The typical function satisfying $(H)$ is $f(t, u, u)=\sum_{i=1}^{n} a_{i}(t) u^{\lambda_{i}}+\sum_{j=1}^{m} b_{j}(t) u^{-\mu_{j}}$, where $a_{i}, b_{j} \in C(0,1), 0<\lambda_{i}<1, \mu_{j}>0,(i=1,2, \ldots, n, j=1,2, \ldots, m)$.

Remark 4.2. Condition $(H)$ includes e-concave function (see [6]) as special case. For example, Liu and Yu [7] consider the existence and uniqueness of positive solution to a class of singular boundary value problem under the following condition:

$$
f\left(t, \lambda u, \frac{v}{\lambda}\right) \geq \lambda^{\alpha} f(t, u, v), \quad \forall u, v>0, \lambda \in(0,1),
$$

where $\alpha \in[0,1)$ and $f(t, u, v)$ is nondecreasing on $u$, nonincreasing on $v$. Clearly, condition $(H)$ is weaker than the above condition (4.1). 
In fact, for any $\lambda \geq 1$, from (4.1) it follows that

$$
f(t, \lambda u, v) \leq f\left(t, \lambda u, \frac{1}{\lambda} v\right) \leq \lambda^{\alpha} f(t, u, v) .
$$

On the other hand, for any $0<\lambda<1$, from (4.1) it follows that

$$
f(t, u, v) \geq f\left(t, \lambda u, \lambda \frac{v}{\lambda}\right) \geq \lambda^{\alpha} f(t, u, \lambda v)
$$

that is, $f(t, u, \lambda v) \leq \lambda^{-\alpha} f(t, u, v)$.

In what follows, by using the results obtained in this paper, we study the boundary value problem

$$
\begin{gathered}
u^{\prime \prime}(t)+\mu t^{-\gamma}(1-t)^{-l}\left(u^{-\alpha}(t)+u^{\beta}(t)+A\right)=0, \quad 0<t<1, \\
u(0)=\sum_{i=1}^{m-2} \alpha_{i} u\left(\eta_{i}\right), \quad u(1)=0,
\end{gathered}
$$

where $\mu>0, \alpha>0, \beta<1, A \geq 0$. We have the following theorem.

Theorem 4.3. A necessary and sufficient condition for problem (4.4) to have smooth positive solution is that

$$
\max \{\gamma+\alpha, l+\alpha, \gamma-\beta, l-\beta, \gamma, l\}<1
$$

Moreover, when the positive solution exists, it is unique.

Remark 4.4. Consider (1.1) and the following singular $m$-point boundary value conditions:

$$
u(0)=0, \quad u(1)=\sum_{i=1}^{m-2} \alpha_{i} u\left(\eta_{i}\right)
$$

By analogous methods, we have the following results.

Assume that $u(t)$ is a $C[0,1]$ positive solution to (1.1) and (4.6), then $u(t)$ can be stated

$$
u(t)=\int_{0}^{1} G(t, s) f(s, u(s), u(s))+\frac{t}{1-\sum_{i=1}^{m-2} \alpha_{i} \eta_{i}} \sum_{i=1}^{m-2} \alpha_{i} \int_{0}^{1} G\left(\eta_{i}, s\right) f(s, u(s), u(s)) d s,
$$

where $G(t, s)$ is defined in (2.4).

Theorem 4.5. Suppose that $(H)$ holds, then a necessary and sufficient condition for the problem (1.1) and (4.6) to have smooth positive solution is that

$$
0<\int_{0}^{1} f(s, s, s) d s<\infty
$$


Theorem 4.6. Suppose $(H)$ and (4.8) hold, then the smooth positive solution to problem (1.1) and (4.6) is also unique $C[0,1]$ positive solution.

\section{Acknowledgment}

Research supported by the National Natural Science Foundation of China (10871116), the Natural Science Foundation of Shandong Province (Q2008A03) and the Doctoral Program Foundation of Education Ministry of China (200804460001).

\section{References}

[1] X. Du and Z. Zhao, "A necessary and sufficient condition of the existence of positive solutions to singular sublinear three-point boundary value problems," Applied Mathematics and Computation, vol. 186, no. 1, pp. 404-413, 2007.

[2] X. Du and Z. Zhao, "On existence theorems of positive solutions to nonlinear singular differential equations," Applied Mathematics and Computation, vol. 190, no. 1, pp. 542-552, 2007.

[3] Z. Wei, "A necessary and sufficient condition for the existence of positive solutions of singular superlinear m-point boundary value problems," Applied Mathematics and Computation, vol. 179, no. 1, pp. 67-78, 2006.

[4] Y. Zhang, "Positive solutions of singular sublinear Emden-Fowler boundary value problems," Journal of Mathematical Analysis and Applications, vol. 185, no. 1, pp. 215-222, 1994.

[5] P. Hartman, Ordinary Differential Equations, Brikhäuser, Boston, Mass, USA, 2nd edition, 1982.

[6] D. J. Guo and V. Lakshmikantham, Nonlinear Problems in Abstract Cones, vol. 5 of Notes and Reports in Mathematics in Science and Engineering, Academic Press, Boston, Mass, USA, 1988.

[7] Y. Liu and H. Yu, "Existence and uniqueness of positive solution for singular boundary value problem," Computers \& Mathematics with Applications, vol. 50, no. 1-2, pp. 133-143, 2005. 